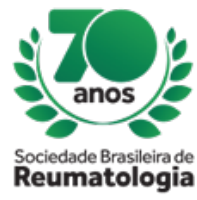

\title{
HYPERTENSIVE EMERGENCY IN A PATIENT WITH LUPUS NEPHRITIS TREATED WITH CYCLOPHOSPHAMIDE: A POSSIBLE COMPLICATION OF THERAPY
}

Keoma Azevedo Sabião (Hospital de Clínicas da Universidade Federal do Paraná, curitiba, PR, Brasil), Salun Coelho Aragão (Hospital de Clínicas da Universidade Federal do Paraná, curitiba, PR, Brasil), Eduardo Santos Paiva (Hospital de Clínicas da Universidade Federal do Paraná, curitiba, PR, Brasil), Maxwell Cassio de Albuquerque Pessoa (Hospital de Clínicas da Universidade Federal do Paraná, curitiba, PR, Brasil), Isadora Welter Pioresan (Hospital de Clínicas da Universidade Federal do Paraná, curitiba, PR, Brasil), Luan Felipe Luckmann (Hospital de Clínicas da Universidade Federal do Paraná, curitiba, PR, Brasil), Nathan Marostica Catolino (Hospital de Clínicas da Universidade Federal do Paraná, curitiba, PR, Brasil), André Luís de Souza Andretta (Hospital de Clínicas da Universidade Federal do Paraná, curitiba, PR, Brasil), Ana Luisa Woidello Miyazima (Hospital de Clínicas da Universidade Federal do Paraná, curitiba, PR, Brasil), Mariana Lechitzki (Hospital de Clínicas da Universidade Federal do Paraná, curitiba, PR, Brasil)

\section{BACKGROUND}

Cyclophosphamide (CYC) is an alkylating immunosuppressive agent, often used in the treatment of systemic lupus erythematosus with severe organ involvement. Among the main adverse effects related to its use we can mention hemorrhagic cystitis, gonadal failure, opportunistic infections and bone marrow suppression. We describe in this report an unusual case of hypertensive emergency in an adult woman after the fourth monthly dose of intravenous CYC as a remission induction therapy for lupus nephritis.

\section{CASE REPORT}

We describe a 24-year-old female patient with a diagnosis of systemic lupus erythematosus with severe renal impairment (class IV lupus nephritis) since 2011, in addition to systemic arterial hypertension. She was refractory to several previous attempts of induction of remission by the renal disease. In November 2018, after new renal flare despite mycophenolate mofetil and tacrolimus, it was suspected of poor therapeutic adherence and opted for replacement of the induction scheme by intravenous CYC. The dose used was $750 \mathrm{mg} / \mathrm{m}^{2}$ in monthly pulses in association with prednisone $0.5 \mathrm{mg} / \mathrm{kg} /$ day. For prevention of hemorrhagic cystitis, it was performed intravenous hydration with $1500 \mathrm{ml}$ of isotonic saline, according to hospital protocol. Initially, patient had a good response of renal disease, but the next day after the fourth CYC infusion, she evolved with sudden visual blurring, nausea and vomiting. On physical examination, she had BP: 190/110 mmhg, in addition to lower limb edema and fundoscopy with findings compatible with acute hypertensive retinopathy. Laboratory tests showed acute renal failure, increased proteinuria and microangiopathic hemolytic anemia. Patient was managed as a hypertensive emergency, evolving with satisfactory clinical and laboratory response.

\section{CONCLUSION}

Hypertensive emergency (HD) is an uncommon complication of arterial hypertension, evidenced by the acute and pronounced elevation of pressure levels, associated with signs of damage to target organs. These may include pulmonary edema, cardiac ischemia, neurological deficits, acute renal failure, aortic dissection, and eclampsia. Considering the potential for morbidity and mortality, it is necessary, besides the management and early recognition of this condition, to investigate the cause of abrupt elevation of blood pressure, thus directing the subsequent treatment. Although not well reported in the literature, and based on the context of the presented clinical case, the hypothesis of direct association of intravenous therapy with CYC and the appearance of HD can be raised. 